\title{
Mitralklappenerkrankung des Hundes im asymptoma- tischen Stadium: Behandeln oder nicht behandeln?
}

\author{
Sonja Schiller
}

\begin{abstract}
Die Mitralklappenendokardiose ist die häufigste Herzerkrankung des Hundes. Oftmals ist sie durch einen jahrelangen präklinischen Verlauf gekennzeichnet und gerade die Behandlung dieses asymptomatischen Stadiums wird selbst in Fachkreisen kontrovers diskutiert. Die folgende Veröffentlichung gibt eine aktuelle Literaturübersicht zur Behandlung der Mitralendokardiose im asymptomatischen Stadium. Sie soll dem Praktiker eine therapeutische Entscheidung nach aktuellstem Wissensstand erleichtern.
\end{abstract}

\section{Relevanz}

Die Mitralklappenendokardiose gilt als die häufigste erworbene Herzerkrankung und stellt ätiologisch die wichtigste Ursache für das Herzversagen des Hundes dar [9]. Sie macht 75\% aller caninen Herzerkrankungen aus [36]. Die Erkrankung zeichnet sich oftmals durch einen jahrelangen präklinischen Verlauf aus, der nicht zwangsläufig im Herzversagen enden muss [7]. Doch gerade die Behandlung dieses asymptomatischen Stadiums wird selbst in Fachkreisen kontrovers diskutiert und nicht einheitlich beantwortet, obwohl es sich für den behandelnden Tierarzt um eine alltägliche Fragestellung handelt.

\section{Klassifikation}

Im Jahr 2009 wurde vom American College of Veterinary Internal Medicine ein Consensus Statement zur Diagnostik und Therapie der Klappenendokardiose herausgegeben. In diesem wurde analog zur Humanmedizin zunächst das Krankheitsstadium des Patienten klassifiziert $[2,21]$. Je nach Stadium wurden entsprechend Leitlinien zur weiteren Diagnostik und Therapie definiert.

\section{Stadium A}

Patienten, bei denen noch keine Herzerkrankung festgestellt werden kann, die aber u.a. einem rassebedingten Erkrankungsrisiko unterliegen, werden in das Stadium A eingeteilt. Dazu gehören beispielsweise Hunde der Rasse Cavalier King Charles Spaniel ohne Herzgeräusch [5]. Das Stadium A gab es in früheren Schemata nicht, es dient der Früherkennung von Herzerkrankungen.

\section{Stadium B}

Dieses Stadium umfasst Patienten, bei denen eine Herzerkrankung objektiv dokumentiert werden kann, die aber aktuell keine Symptome eines Herzversagens zeigen. Dies wären z. B. Dackel mit einem Herzgeräusch, bei denen in der Echokardiografie eine Mitralklappenendokardiose diagnostiziert werden kann.

\section{Eine Leistungsschwäche darf nicht mit einem Herzversagen (Lungenödem, Aszites, Thoraxerguss) gleichgesetzt werden.}

Husten kann häufig auch aufgrund tracheobronchialer Erkrankungen auftreten und muss klar von einem Herzversagen differenziert werden [2].

Unterschieden wird in der Gruppe B das Substadium B1, bei dem keine Kompensationsanzeichen und somit keine Herzvergrößerung vorliegen, sowie das Substadium B2 mit einer röntgenologisch oder echokardiografisch dokumentierten Kardiomegalie. Zwischen dem Stadium B1 und B2 herrschen klare prognostische und therapeutische Unterschiede. Das Hauptaugenmerk dieses Artikels gilt der Therapie der Mitralklappenendokardiose im Stadium B2.

\section{Stadium C}

Dem Stadium C werden Patienten, die aktuell oder früher ein objektiv dokumentiertes Herzversagen (Lungenödem, Aszites, Thoraxerguss) erfahren haben, zugeordnet. Je nach aktueller Klinik unterscheidet man Patienten, die medikamentös ambulant behandelt werden können und Patienten, die aufgrund hochgradiger lebensbedrohlicher Symptome einer Herzinsuffizienz eine stationäre Therapie benötigen.

\section{Stadium D}

Dem Stadium D gehören schließlich Patienten mit refraktären Symptomen einer Herzinsuffizienz trotz kardialer Standardtherapie an.

\section{Therapieempfehlungen}

Die moderne Kardiologie der letzten 10-15 Jahre orientiert sich streng an den zur Verfügung stehenden Veröffentlichungen. So werden heutzutage Änderungen von bestehenden Therapieempfehlungen nur noch gemäß prospektiver, placebokontrollierter, doppelt geblindeter klinischer Studien mit signifikanter Auswirkung auf die Morbidität und Mortalität akzeptiert [3]. Dies wird vor allem in der im Consensus Statement kontrovers diskutierten Therapie der Mitralklappenendokardiose im Stadium B2 deutlich.

\section{Stadium B1}

Ein Konsens besteht lediglich zum Stadium B1, in dem noch keine hämodynamischen Auswirkungen mit Kardiomegalie bestehen [2]. Das Komitee empfiehlt bei Hunden mit Mitralendokardiose im Stadium B1 keine medikamentöse Therapie, aber Kontrolluntersuchungen im mindestens 12-monatigen Abstand. Zugrunde liegen müssen jedoch [8]:

- eine normale Blutdruckmessung 
- ein unauffälliges Basislabor (Hämatokrit, Eiweiß, Kreatinin, Urinuntersuchung)

- ein normales Thoraxröntgen ohne Kardiomegalie nach dem Vertebral Heart Score (VHS)

Zu Letzterem existieren mittlerweile rassespezifische Größenangaben ( $\triangleright$ Tab. 1), v.a. für breitbrüstige Hunderassen und Windhunde $[4,22,25,27,31]$. Der von Buchanan und Bücheler genannte Referenzwert von 9,7 $\pm 0,5$ Brustwirbelkörpern ist zur Orientierung aber sicher noch von Bedeutung [8]. Die Herzgröße und die zugrunde liegende Herzerkrankung sollten möglichst echokardiografisch kontrolliert werden.

\section{Stadium B2}

Dem Stadium B2 werden Patienten mit einer röntgenologisch und echokardiografisch dokumentierten Kardiomegalie zugeordnet [2]. Die verringerte Aus-

Tab. 1 Rassespezifische Referenzwerte für die rechts anliegende VHS-Messung.

\begin{tabular}{|c|c|}
\hline Rasse & $\begin{array}{l}\text { Median } \pm \\
\text { Standard- } \\
\text { abweichung }\end{array}$ \\
\hline Beagle & $10,3 \pm 0,4[25]$ \\
\hline Boston Terrier & $11,7 \pm 1,4[22]$ \\
\hline Boxer & $11,6 \pm 0,8[27]$ \\
\hline Bulldogge & $12,7 \pm 1,7[22]$ \\
\hline $\begin{array}{l}\text { Cavalier King Charles } \\
\text { Spaniel }\end{array}$ & $10,6 \pm 0,7[27]$ \\
\hline Dackel & $9,7 \pm 0,5[22]$ \\
\hline $\begin{array}{l}\text { Deutscher Schäfer- } \\
\text { hund }\end{array}$ & $9,7 \pm 0,7[27]$ \\
\hline Dobermann & $10,0 \pm 0,6[27]$ \\
\hline Greyhound & $10,5 \pm 0,1[31]$ \\
\hline Labrador & $10,8 \pm 0,6[27]$ \\
\hline Lhasa Apso & $9,6 \pm 0,8[22]$ \\
\hline Mops & $10,7 \pm 0,9[22]$ \\
\hline Rottweiler & $9,8 \pm 0,1[31]$ \\
\hline Shi Tzu & $9,5 \pm 0,6[22]$ \\
\hline Whippet & $11,3 \pm 0,5[4]$ \\
\hline Yorkshire Terrier & $9,9 \pm 0,6[22]$ \\
\hline Zwergspitz & $10,5 \pm 0,9[22]$ \\
\hline
\end{tabular}

wurfleistung durch die hochgradige Mitralinsuffizienz führt zur Aktivierung neurohumoraler Kompensationsmechanismen (Erhöhung des Sympathikotonus und Aktivierung des Renin-AngiotensinAldosteron-Systems, RAAS). Dies zieht auf Dauer negative Umbauprozesse am Herzen, das sogenannte Remodeling, nach sich [35].

\section{Blockade der Kompensations- mechanismen}

Eine medikamentöse Blockade neurohumoraler Kompensationsmechanismen stellt in der Theorie einen Ansatzpunkt zur Verlangsamung des Erkrankungsverlaufs dar. Jedoch konnte bisher keine Studie einen signifikanten Vorteil einer medikamentösen Therapie verglichen zum Placebo bis zum Zeitpunkt des Auftretens eines Herzversagens zeigen.

Zu den bisher untersuchten Medikamenten gehören:

- ACE-Hemmer (Enalapril, Benazepril)

- Aldosteronantagonisten (Spironolacton)

- Kalziumsensitizer und Phosphodiesterasehemmer (Pimobendan)

- $\beta$-Blocker (Carvedilol)

Aktuell laufen noch verschiedene Studien (EPIC, DELAY), deren Ergebnisse mit Spannung erwartet werden. Die Studiendesigns werden weiter unten beschrieben.

\section{ACE-Hemmer}

Hintergrund zum Einsatz eines ACEHemmers ist die Blockade des ReninAngiotensin-Aldosteron-Systems, das durch die verringerte Auswurfleistung und damit durch die verringerte Perfusion der Nieren aktiviert wird [35]. Renin bildet aus Angiotensinogen Angiotensin I, das wiederum vom Angiotensinconverting-Enzyme (ACE) zu Angiotensin II umgewandelt wird. Angiotensin II wirkt vasokonstriktorisch und führt zur Freisetzung von Aldosteron aus der Nebennierenrinde und somit zur vermehrten Salz- und Wasserrückresorption in der Niere. Vorlast und Blutdruck steigen, womit die renale Perfusion erhalten bleibt, der Herzmuskel jedoch durch die erhöhte Vor- und Nachlast chronisch geschädigt wird ( $\triangleright$ Abb. 1) [44]. Aldosteron führt zusätzlich zu einer Fibrose am Myokard [12, 46].

Der im Jahr 2002 publizierte SVEP-Trial (Scandinavian Veterinary Enalapril Project) ist eine prospektive, randomisierte, doppelt geblindete, placebo-kontrollierte Multicenterstudie [26]. Sie schloss 229 Cavalier King Charles Spaniel mit Mitralendokardiose im asymptomatischen Stadium B ein, die mit einem ACEHemmer (Enalapril 0,25-0,5 mg/kg 1× täglich) oder einem Placebo behandelt wurden. Es konnte kein signifikanter Nutzen in der Zeitspanne bis zum Auftreten eines Herzversagens gezeigt werden, auch nicht in der Subpopulation der Patienten mit Kardiomegalie (Stadium B2). Aus diesem Grund wird der Einsatz eines ACE-Hemmers im asymptomatischen Stadium von der Studiengruppe nicht empfohlen. Da nur Hunde der Rasse Cavalier King Charles Spaniel an der Studie beteiligt waren, stellte sich jedoch die Frage, ob die Studienergebnisse auch auf andere Rassen übertragbar sind.

Die VETPROOF-Studie (Veterinary Enalapril Trial to Prove Reduction in Onset of Failure), ebenfalls eine prospektive Doppelblindstudie, schloss 124 Hunde aller Rassen mit kompensierter Mitralendokardiose im Stadium B2 ein. Auch sie stellte die Behandlung mit Enalapril (0,23-0,66 mg/kg $1 \times$ täglich) und einem Placebo gegenüber [1]. Das primäre Studienziel - der Zeitpunkt bis zum Eintreten des Herzversagens - zeigte keinen signifikanten Unterschied zwischen den Gruppen (895 Tage Enalapril versus 778 Tage Placebo). In der Subanalyse zeigte sich jedoch ein signifikanter Unterschied zugunsten von Enalapril in der Gesamtmortalität, der Zahl der Hunde ohne Herzversagen an Tag 500 und 1500 sowie der herzversagensfreien Tage.

Atkins propagiert aus diesem Grund den Einsatz eines ACE-Hemmers bei Patienten mit kompensierter Mitralendokardiose im Stadium B2 [3]. Die Forschungsgruppe um Valerie Chetboul betont dies ebenfalls in einer retrospektiven Studie. Diese zeigt einen signifikanten Nutzen von Benazepril in der Gesamtüberlebenszeit sowie in der Zeit bis zum Eintritt eines kardialen Ereignisses (Herz- 


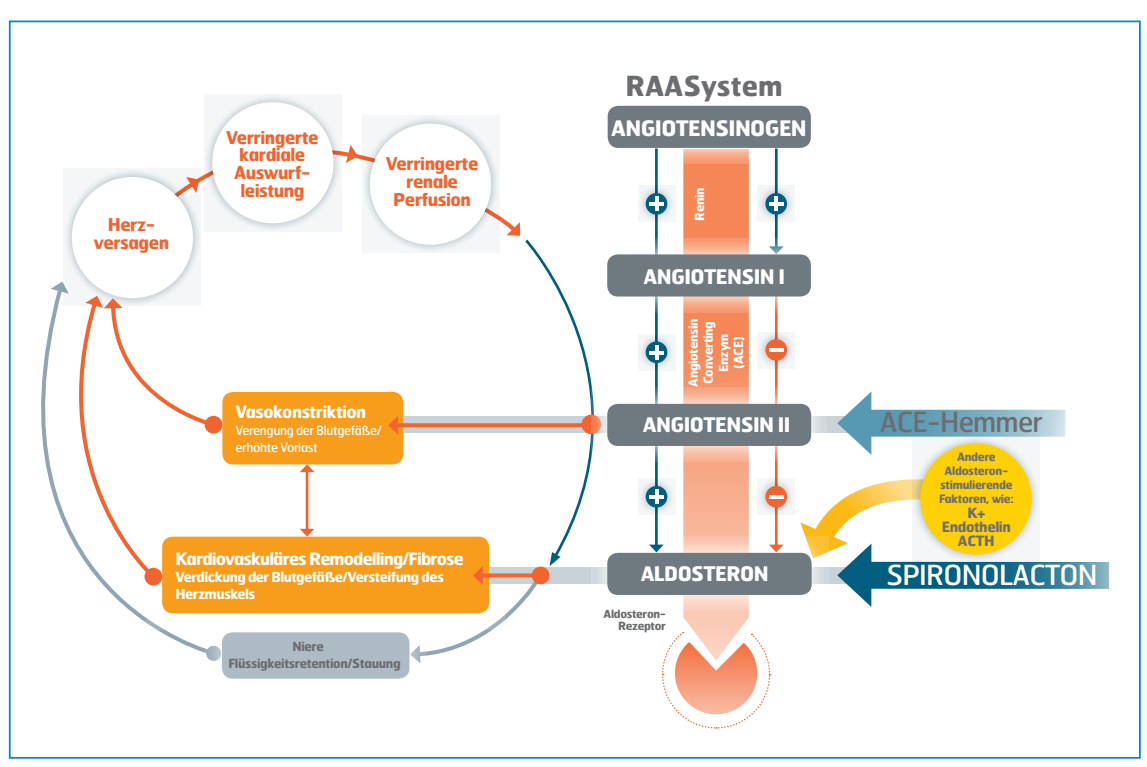

Abb. 1 Darstellung des Renin-Angiotensin-Aldosteron-Systems (RAAS-System) und dessen Auswirkung auf das kardiovaskuläre System im Herzversagen. Mögliche Ansatzpunkte zur Blockade des RAAS-Systems durch medikamentöse Therapie: Ein ACE-Hemmer verhindert die Umwandlung von Angiotensin I zu Angiotensin II. Spironolacton blockiert die Aldosteron-Rezeptoren und somit die schädigende Wirkung von Aldosteron. (C) Ceva Tiergesundheit GmbH

versagen, plötzlicher Herztod) bei Hunden anderer Rassen als dem Cavalier King Charles Spaniel [39]. Kittleson zweifelt die Aussagekraft der statistischen Analyse dieser retrospektiven Studie jedoch an [23] und gehört mit Häggström zu den ACE-Hemmer-Gegnern. Dies beruht auch auf der Tatsache, dass Häggström keine erhöhten Renin- oder Aldosteronspiegel im Plasma bei Patienten vor Auftreten eines Herzversagens messen konnte [14, 15]. Andere Forschungsgruppen wiederum berichten sehr wohl von erhöhten Plasmaspiegeln [37].

Zusammenfassend lässt sich sagen, dass keine Studie bisher eindeutig beweisen konnte, dass die Gabe eines ACE-Hemmers bei Patienten mit kompensierter Mitralendokardiose zu einer statistisch signifikanten Verlangsamung des Krankheitsverlaufs führt. Dennoch bestehen Hinweise auf positive Effekte in der Krankheitsentwicklung.

\section{Aldosteronantagonisten}

Wirkstoffe wie Spironolacton werden aufgrund ihrer schwach diuretischen und antifibrotischen Eigenschaften bei Patienten mit Herzinsuffizienz eingesetzt [34].
Interessanterweise lässt sich die Aldosteronproduktion durch Blockade des RAAS-Systems mittels eines ACE-Hemmers nur vorübergehend supprimieren, da auch andere Enzyme (Chymase, Cathepsin G) in der Lage sind, Angiotensin II unabhängig vom ACE zu bilden. Des Weiteren können bestimmte endogene Faktoren wie der Kaliumspiegel, Endothelin oder ACTH (unabhängig von Angiotensin II) die Aldosteron-Synthese anregen ( $\triangleright$ Abb. 1). Diese Phänomene erklären eine Erhöhung des Aldosteronspiegels trotz bestehender Therapie mit einem ACE-Hemmer (Aldosteron-Escape). Das Aldosteron-Escape-Phänomen bildet die Grundlage zum Einsatz von Aldosteronantagonisten, auch in Kombination mit ACE-Hemmern [14, 28, 38].

Die Studie von Bernay zeigte, dass der Einsatz von Spironolacton zusätzlich zur Gabe von ACE-Hemmern und zur Entwässerung bei Patienten mit einer symptomatischen Mitralendokardiose zu einer 69\%igen Reduktion der kardialen Mortalität führt [6]. Jedoch wurde bei der Studie nicht klar zwischen Patienten im Stadium B2 und C unterschieden. Nur wenige der eingeschlossenen Hunde erreichten zudem den kardialen Studienendpunkt. Dies zog berechtigte Kritik aus den Schlussfolgerungen der Studie nach sich [24]. Trotzdem befürwortet die Mehrheit der Kardiologen die zusätzliche Gabe von Spironolacton im symptomatischen Stadium C, obwohl die Studie von Bernay zu diesem Zeitpunkt noch nicht erschienen war [2].

Die Mehrheit der Kardiologen empfehlen Spironolacton zur kardialen TripleTherapie (Furosemid, Pimobendan und ACE-Hemmer) im Stadium C hinzuzufügen.

Hezzel führte 2012 eine randomisierte, placebokontrollierte Doppelblind-Pilotstudie an 20 Hunden durch [19]. Alle Tiere litten an einer Mitralendokardiose im Stadium B. Dabei konnte gezeigt werden, dass die Gabe von Spironolacton gegenüber einem Placebo zu einem geringeren Anstieg von:

- NT-proBNP (kardiale Biomarker als Maß für eine Volumenbelastung)

- LA/Ao (Verhältnis linkes Atrium/Aorta als Maß für eine linksatriale Vergrößerung [18])

- LVEDD (linksventrikulärer Innendurchmesser in der Diastole)

innerhalb von 6 Monaten führt.

NT-proBNP, LA/Ao und LVEDD stellen Parameter für eine Herzvergrößerung dar. Die Studie wird momentan fortgeführt, um größere Fallzahlen zu erreichen.

Spironolacton ist gut verträglich, wie Lefebvre in einer Doppelblindstudie an 169 Hunden mit Mitralendokardiose beweisen konnte [29]. Eine Langzeitdosierung von $2 \mathrm{mg} / \mathrm{kg} 1 \times$ täglich alleine oder in Kombination mit anderen kardialen Medikamenten (ACE-Hemmer, Furosemid, Digoxin) führt nicht zu einem erhöhten Risiko von unerwünschten Nebenwirkungen - insbesondere nicht zu Elektrolytverschiebungen, Hyperkaliämie sowie Nierenerkrankungen. In dieser Studie wurde eine höhere Zahl von Todesfällen durch Herz- und/oder Nierenerkrankungen in der Placebogruppe festgestellt. Aufgrund dieser Befunde wird neuerdings eine renoprotektive Wirkung von Spironolacton diskutiert. 
Mit großer Spannung werden die Ergebnisse der DELAY-Studie (DELay the Appearance for sYmptoms of canine degenerative mitral valve disease treated with Spironolactone and Benazepril) erwartet. In der über 3,5 Jahre laufenden Multicenterstudie (Laufzeit bis 2015) soll anhand von 240 Hunden mit Mitralendokardiose im Stadium B2 die Effizienz von Benazepril in Kombination mit Spironolacton bis zum Auftreten eines Herzversagens evaluiert werden. Benazepril und Spironolacton stehen der Veterinärmedizin bereits als Kombinationspräparat zur Verfügung, was die Besitzercompliance durch Reduktion der Tablettenanzahl verbessert.

\section{Kalziumsensitizer und Phosphodiesterasehemmer}

Der Kalziumsensitizer und Phosphodiesterasehemmer Pimobendan wirkt positiv inotrop und vasodilatatorisch und ist seit Erscheinen der QUEST-Studie (Quality of Life and Extension of Survival Time, 2008) in der kardialen Herzversagenstherapie indiziert [16]. Die Studie konnte an 260 Patienten mit Mitralendokardiose im Herzversagen zeigen, dass die Überlebenszeit verglichen $\mathrm{zu}$ einem ACEHemmer signifikant verlängert wird (267 Tage in der Pimobendan-Gruppe versus 140 Tage in der Benazepril-Gruppe), $[16,17]$.

Hintergrund des positiven Effekts ist neben der Vasodilatation die Tatsache, dass bei Mitralendokardiosepatienten trotz Hyperkinesie ebenfalls eine systolische Dysfunktion mit multiplen Myokardinfarkten vorliegt [45]. Pimobendan ist ab dem Auftreten eines Herzversagens (Stadium C) definitiv angezeigt und mittlerweile auch als i.v.-Injektionslösung zugelassen.

Zur Anwendung von Pimobendan im asymptomatischen Stadium B wurden bisher nur 2 Studien veröffentlicht. Chetboul [10] untersuchte in einer randomisierten Blindstudie an 12 Beaglen mit milder asymptomatischer Mitralendokardiose im Stadium B1 die Behandlung mit Pimobendan versus Benazepril über 512 Tage. Die Hunde wurden echokardiografisch und nach Beendigung der Studie auch histopathologisch untersucht. Die Forschungsgruppe fand eine Zunahme der systolischen Funktion, des mitralen Regurgitationsjets und der histologischen Klappenläsionen verglichen zur Benazepril-Gruppe.

Zum Stadium B2 hat Ouellet [33] eine prospektive, geblindete Studie an 24 Hunden, die neben einem ACE-Hemmer Pimobendan versus einem Placebo über einen Zeitraum von 6 Monaten erhielten, veröffentlicht. Das Ergebnis der Studie zeigte keinen Nutzen durch den zusätzlichen Einsatz von Pimobendan auf die systolische Funktion oder das Regurgitationsvolumen.

Aufgrund der vorliegenden Studien wird von einer Behandlung mit Pimobendan im Stadium B abgeraten.

Die Durchführer der EPIC-Studie (Evaluating Pimobendan In Cardiomegaly) hoffen beweisen zu können, dass der Zeitpunkt der Entwicklung eines Herzversagens durch die Gabe von Pimobendan hinausgezögert wird. In der Studie, einer doppelt geblindeten, randomisierten, placebokontrollierten Multicenterstudie in 11 Ländern, die noch bis 2015 läuft, werden 360 kleine Hunde mit Mitralendokardiose im Stadium B2 über einen Zeitraum von mindestens 3 Jahren mit Pimobendan (180 Hunde) oder einem Placebo (180 Hunde) behandelt. Studienendpunkt ist die Entwicklung eines kardialen Herzversagens (Lungenödem) oder der kardiale Tod.

Bis zur Veröffentlichung der Ergebnisse wird aufgrund der vorliegenden Studien noch vom Einsatz von Pimobendan bei Hunden mit asymptomatischer Mitralendokardiose abgeraten.

\section{$\beta$-Blocker}

Zuletzt besteht eine wohl nie enden wollende Diskussion über den Einsatz von $\beta$-Blockern. Eine chronische Sympathikusaktivierung, wie sie bei Herzerkrankungen vorhanden ist, führt durch Vasokonstriktion, myokardiale Ischämie und Arrhythmien zu einer weiteren Schädigung des Herzens. In der Humanmedizin gehören ß-Blocker wie Carvedilol zur kardialen Standardtherapie mit signifikanter Reduktion der kardialen Mortalität [21]. Prospektive kontrollierte Studien fehlen hierzu in der Tiermedizin oder sind auf kleine Fallzahlen ohne signifikanten Nutzen beschränkt [30]. Ihre Gabe wird derzeit im asymptomatischen Stadium der Mitralinsuffizienz nicht empfohlen. Patienten mit Rhythmusstörungen sind in dieser Empfehlung nicht enthalten.

\section{Herzglykoside}

Digoxin wurde früher vor allem aufgrund seiner positiv inotropen Wirkung eingesetzt. Mittlerweile wird es aufgrund der wesentlich potenteren Wirkung neuerer Wirkstoffe (Pimobendan) abgelöst. Es hat in der Behandlung der asymptomatischen Mitralendokardiose derzeit keine Indikation und wird praktisch nur noch aufgrund seiner negativ chronotropen und dromotropen Wirkung bei der Behandlung von Vorhofflimmern eingesetzt [13]. Fehlende Studien tragen vermutlich aber auch zu dieser Empfehlung bei.

\section{Fazit}

Nach Meinung der Autorin ist es nach derzeitiger Studienlage vertretbar, im Stadium B2 der Mitralendokardiose sowohl einen ACE-Hemmer und Spironolacton (oder das Kombinationspräparat) einzusetzen als auch nicht zu therapieren. Persönliche Grundeinstellungen der Besitzer können möglicherweise die Entscheidung beeinflussen.

\section{.konkret}

Eine korrekte Diagnose und Klassifizierung des Patienten sind unerlässlich, um ein drohendes Herzversagen frühzeitig erkennen zu können.

Die durchschnittliche Zeit von Mitralinsuffizienzpatienten bis zur Entwicklung eines Herzversagens beträgt $27 \mathrm{Mo-}$ nate $[1,7,26]$. Die Erkrankung ist dabei durch einen nicht linearen Verlauf charakterisiert [43]. 
Negativ prognostische Parameter sind [7]:

- die Lautstärke des Herzgeräuschs

- Husten

- die linksventrikuläre und die linksatriale Größe

- ein erhöhter mitraler Einstrom [7]

Beim Vorliegen negativ prognostischer Parameter sind häufigere echokardiografische und röntgenologische Kontrollen des Patienten indiziert. Diese sollten etwa alle 3-6 Monate erfolgen. Zur Detektion eines beginnenden Lungenödems kann es hilfreich sein, den Besitzer regelmäßig die Atemfrequenz in Ruhe bestimmen zu lassen $[41,42]$, die dann in der Regel 40 Atemzüge/Minute überschreitet.

Ein erhöhtes NT-proBNP (>1500 pmol/l) oder ein progressiv ansteigender Wert stellen einen Risikofaktor für die Entstehung eines Lungenödems innerhalb von 12 Monaten dar [11, 32, 40]. Dies sollte den Kliniker und Kardiologen zu einer engmaschigen Kontrolle des Patienten anhalten, um ihm so die Situation eines lebensbedrohlichen Lungenödems zu ersparen.

Möglicherweise werden die Ergebnisse der DELAY- und der EPIC-Studie dazu beitragen, neue, klarere Leitlinien für das Stadium B2 zu formulieren.

Online zu finden unter

http://dx.doi.org/10.1055/s-0034-1384499

\section{Literatur}

1 Atkins C, Keene BW, Brown WA. Results of the veterinary enalapril trial to prove reduction in onset of heart failure in dogs chronically treated with enalapril alone for compensated, naturally occurring mitral valve insufficiency. J Am Vet Med Assoc 2007; 231 (7): 1061-1069

2 Atkins $C$, Bonagura J, Ettinger $S$ et al. Guidelines for the diagnosis and treatment of canine chronic valvular heart disease. J Vet Intern Med 2009; 23 (6): 1142-1150

3 Atkins C, Häggström J. Pharmacologic management of myxomatous mitral valve disease in dogs. J Vet Cardiol 2012; 14 (1): 165-184
4 Bavegems V, Van Caelenberg A, Duchateau L et al. Vertebral heart size ranges specific for whippets. Vet Radiol Ultrasound 2005; 46 (5): 400-403

5 Beardow AW, Buchanan JW. Chronic mitral valve disease in cavalier King Charles spaniels: 95 cases (1987-1991). J Am Vet Med Assoc 1993; 203 (7): 1023-1029

6 Bernay F, Bland JM, Häggström J et al. Efficacy of spironolactone on survival in dogs with naturally occurring mitral regurgitation caused by myxomatous mitral valve disease. J Vet Intern Med 2010; 24 (2): 331-341

7 Borgarelli M, Crosara S, Lamb K et al. Survival characteristics and prognostic variables of dogs with preclinical chronic degenerative mitral valve disease attributable to myxomatous degeneration. J Vet Intern Med 2012; 26 (1): 69-75

8 Buchanan JW, Bücheler J. Vertebral scale system to measure canine heart size in radiographs. J Am Vet Med Assoc 1995; 206 (2): 194-199

9 Buchanan J. Prevalence of cardiovascular disorders. In: Textbook of Canine and Feline
Cardiology. Fox PR, Sisson DD, Moise N, eds. Philadelphia: Saunders; 1999

10 Chetboul V, Lefebvre HP, Sampedrano CC et al. Comparative adverse cardiac effects of pimobendan and benazepril monotherapy in dogs with mild degenerative mitral valve disease: a prospective, controlled, blinded, and randomized study. J Vet Intern Med 2007; 21 (4): 742-253

11 Chetboul V, Serres F, Tissier R et al. Association of plasma N-terminal pro-B-type natriuretic peptide concentration with mitral regurgitation severity and outcome in dogs with asymptomatic degenerative mitral valve disease. J Vet Intern Med 2009; 23 (5): 984-994

12 Farquharson CA, Struthers AD. Aldosterone induces acute endothelial dysfunction in vivo in humans: evidence for an aldosteroneinduced vasculopathy. Clin Sci (Lond) 2002; 103 (4): 425-431

13 Gelzer AR, Kraus MS, Rishniw M et al. Combination therapy with digoxin and diltiazem controls ventricular rate in chronic atrial fibrillation in dogs better than digoxin or diltiazem monotherapy: a randomized 
crossover study in 18 dogs. J Vet Intern Med 2009; 23 (3): 499-508

14 Häggström J, Hansson K, Karlberg BE et al. Effects of long-term treatment with enalapril or hydralazine on the renin-angiotensinaldosterone system and fluid balance in dogs with naturally acquired mitral valve regurgitation. Am J Vet Res 1996; 57 (11): 1645-1652

15 Häggström J, Hansson K, Kvart C et al. Effects of naturally acquired decompensated mitral valve regurgitation on the renin-angiotensinaldosterone system and atrial natriuretic peptide concentration in dogs. Am J Vet Res 1997; 58 (1): 77-82

16 Häggström J, Boswood A, O’Grady M et al. Effect of pimobendan or benazepril hydrochloride on survival times in dogs with congestive heart failure caused by naturally occurring myxomatous mitral valve disease: the QUEST study. J Vet Intern Med 2008; 22 (5): 1124-1235

17 Häggström J, Boswood A, O’Grady M et al. Longitudinal analysis of quality of life, clinical, radiographic, echocardiographic, and laboratory variables in dogs with myxomatous mitral valve disease receiving pimobendan or benazepril: the QUEST study. J Vet Intern Med 2013; 27 (6): 1441-1451

18 Hansson K, Häggström J, Kvart C et al. Left atrial to aortic root indices using two-dimensional and $\mathrm{M}$-mode echocardiography in cavalier King Charles spaniels with and without left atrial enlargement. Vet Radiol Ultrasound 2002; 43 (6): 568-575

19 Hezzell MJ, Boswood A, Elliott J. Treatment of dogs with compensated degenerative mitral valve disease (DMVD) with spironolactone. Maastricht: ECVIM-CA Congress; 2012. Im Internet: http://www.vin.com/Members/ Proceedings/Proceedings.plx?CID=ECVIM201 2\&PID=pr84851\&O=Generic; Stand: 11.2014

20 Hungerbühler S, Henrich E. Die Mitralklappenendokardiose - aktueller Stand der Diagnose und Therapie. Kleintierprax 2014; 59 (6): 329-352

21 Hunt SA, Abraham WT, Chin MH et al. Focused update incorporated into the ACC/ AHA 2005. Guidelines for the diagnosis and management of heart failure in adults. A report of the American College of Cardiology Foundation/American Heart Association Task Force on Practice Guidelines developed in collaboration with the International Society for Heart and Lung Transplantation. J Am Coll Cardiol 2009; 53 (15): e1-e90

22 Jepsen-Grant K, Pollard RE, Johnson LR. Vertebral heart scores in eight dog breeds. Vet Radiol Ultrasound 2013; 54 (1): 3-8

23 Kittleson M, Rishniw M, Pion P et al. Effect of benazepril on survival and cardiac events in dogs with asymptomatic mitral valve disease: a retrospective study of 141 cases. J Vet Intern Med 2009; 23 (3): 445-446; author reply $447-448$
24 Kittleson MD, Bonagura JD. Re: Efficacy of spironolactone on survival in dogs with naturally occurring mitral regurgitation caused by myxomatous mitral valve disease. J Vet Intern Med 2010; 24 (6): 1245-1256; author reply 1247-1248

25 Kraetschmer S, Ludwig K, Meneses F et al. Vertebral heart scale in the beagle dog. J Small Anim Pract 2008; 49 (5): 240-243

26 Kvart C, Häggström J, Pedersen HD et al. Efficacy of enalapril for prevention of congestive heart failure in dogs with myxomatous valve disease and asymptomatic mitral regurgitation. J Vet Intern Med 2002; 16 (1): 80-88

27 Lamb CR, Wikeley H, Boswood A et al. Use of breed-specific ranges for the vertebral heart scale as an aid to the radiographic diagnosis of cardiac disease in dogs. Vet Rec 2001; 148 (23): 707-711

28 Lantis AC, Atkins CE, DeFrancesco TC. Aldosterone escape in furosemide-activated circulating renin-angiotensin-aldosterone system (RAAS) in normal dogs. J Vet Intern Med 2010; 24: 672

29 Lefebvre HP, Olliver E, Atkins CE et al. Safety of spironolactone in dogs with chronic heart failure because of degenerative valvular disease: a population-based, longitudinal study. J Vet Intern Med 2013; 27 (5): 1083-1091

30 Marcondes-Santos M, Tarasoutchi F, Mansur AP et al. Effects of carvedilol treatment in dogs with chronic mitral valvular disease. J Vet Intern Med 2007; 21 (5): 996-1001

31 Marin LM, Brown J, McBrien C et al. Vertebral heart size in retired racing Greyhounds. Vet Radiol Ultrasound 2007; 48 (4): 332-334

32 Moonarmart W, Boswood A, Luis Fuentes V et al. N-terminal pro B-type natriuretic peptide and left ventricular diameter independently predict mortality in dogs with mitral valve disease. J Small Anim Pract 2010; 51 (2): 84-96

33 Ouellet M, Bélanger MC, Difruscia R et al. Effect of pimobendan on echocardiographic values in dogs with asymptomatic mitral valve disease. J Vet Intern Med 2009; 23 (2): 258-263

34 Ovaert P, Elliott J, Bernay F et al. Aldosterone receptor antagonists - how cardiovascular actions may explain their beneficial effects in heart failure. J Vet Pharmacol Ther 2010; 33 (2): 109-117

35 Oyama MA. Neurohormonal activation in canine degenerative mitral valve disease: implications on pathophysiology and treatment. J Small Anim Pract 2009; 50 (Suppl 1): 3-11

36 Oyama MA, Levy RJ. Insights into serotonin signaling mechanisms associated with canine degenerative mitral valve disease. J Vet Intern Med 2010; 24 (1): 27-36
37 Pedersen HD, Koch J, Poulsen K et al. Activation of the renin-angiotensin system in dogs with asymptomatic and mildly symptomatic mitral valvular insufficiency. J Vet Intern Med 1995; 9 (5): 328-331

38 Pitt B. „Escape“ of aldosterone production in patients with left ventricular dysfunction treated with an angiotensin converting enzyme inhibitor: implications for therapy. Cardiovasc Drugs Ther 1995; 9 (1): 145-149

39 Pouchelon JL, Jamet N, Gouni V et al. Effect of benazepril on survival and cardiac events in dogs with asymptomatic mitral valve disease: a retrospective study of 141 cases. J Vet Intern Med 2008; 22 (4): 905-914

40 Reynolds CA, Brown DC, Rush JE et al. Prediction of first onset of congestive heart failure in dogs with degenerative mitral valve disease: the PREDICT cohort study. J Vet Cardiol 2012; 14 (1): 193-202

41 Schober KE, Hart TM, Stern JA et al. Detection of congestive heart failure in dogs by Doppler echocardiography. J Vet Intern Med 2010; 24 (6): 1358-1368

42 Schober KE, Hart TM, Stern JA et al. Effects of treatment on respiratory rate, serum natriuretic peptide concentration, and Doppler echocardiographic indices of left ventricular filling pressure in dogs with congestive heart failure secondary to degenerative mitral valve disease and dilated cardiomyopathy. J Am Vet Med Assoc 2011; 239 (4): 468-479

43 Tarnow I, Olsen LH, Kvart C et al. Predictive value of natriuretic peptides in dogs with mitral valve disease. Vet J 2009; 180 (2): 195-201

44 Unger T. The role of the renin-angiotensin system in the development of cardiovascular disease. Am J Cardiol 2002; 89 (2A): 3A-9A; discussion 10A

45 Urabe Y, Mann DL, Kent RL et al. Cellular and ventricular contractile dysfunction in experimental canine mitral regurgitation. Circ Res 1992; 70 (1): 131-147

46 Weber KT, Brilla CG. Pathological hypertrophy and cardiac interstitium. Fibrosis and renin-angiotensin-aldosterone system. Circulation 1991; 83 (6): 1849-1865

\section{Dr. Sonja Schiller}

Fachtierärztin für Innere Medizin der Kleintiere,

Zusatzbezeichnung Kardiologie

Mitglied des Collegium Cardiologicum

Mobile Kleintier-Kardiologie

Alleestr. 3

65439 Flörsheim

www.kleintier-kardiologie.de

kontakt@kleintier-kardiologie.de 